\title{
Clinical Efficacy of Prodom-Assisted Urokinase in the Treatment of Male Infertility Caused by Impaired Semen Liquefaction
}

\author{
Kaiyi Mao, ${ }^{1}$ Zongping Chen ${ }^{D},{ }^{1}$ Mengzhi Li, ${ }^{1}$ Chengren Gou, ${ }^{1}$ Zidong Zhou, ${ }^{1}$ Yong Yan, \\ Chao Chen, ${ }^{2}$ Tong Liu, ${ }^{1}$ Chenghong Zou, ${ }^{3}$ Yuhong Yao, ${ }^{3}$ and $\mathrm{Xu} \mathrm{Li}^{3}$ \\ ${ }^{1}$ Department of Urology, Affiliated Hospital of Zunyi Medical University, Zunyi, Guizhou 563000, China \\ ${ }^{2}$ Department of Urology, Beijing Shijitan Hospital, Capital Medical University, Beijing 100038, China \\ ${ }^{3}$ Department of Urology, The Second Affiliated Hospital of Zunyi Medical University, Zunyi, Guizhou 563000, China \\ Correspondence should be addressed to Zongping Chen; 2404259310@qq.com
}

Received 14 September 2020; Revised 9 December 2020; Accepted 31 December 2020; Published 20 January 2021

Academic Editor: Youlang Zhou

Copyright () 2021 Kaiyi Mao et al. This is an open access article distributed under the Creative Commons Attribution License, which permits unrestricted use, distribution, and reproduction in any medium, provided the original work is properly cited.

\begin{abstract}
Purpose. To evaluate the clinical efficacy of prodom in the administration of urokinase in the vagina in couples with impaired semen liquefaction. Materials and Methods. Overall, 261 patients with impaired semen liquefaction were randomly divided into prodom-assisted urokinase treatment (PAUT) group $(n=91)$, syringe-assisted urokinase treatment (SAUT) group $(n=86)$, and traditional treatment (TT) group $(n=84)$ in the first stage. If the first stage of treatment failed, other treatment methods were initiated instead and the patients were grouped according to the newer treatment method in the second stage. The pregnancy rate, time-to-conception, and treatment costs were evaluated in each group. Results. In the first stage, the pregnancy rate in the PAUT, SAUT, and TT groups was $69.23 \%, 29.07 \%$, and $22.62 \%$, respectively; the time-to-conception was $2.66 \pm 1.44,3.69 \pm 2.61$, and $3.86 \pm 3.00$ months, respectively; the treatment costs were $658.18 \pm 398.40,666.67 \pm 507.50$, and $680.56 \pm 480.94 \$$, respectively. The pregnancy rate and time-to-conception were different in the PAUT group compared with those in SAUT and TT groups (all $P<0.05)$. However, the difference in treatment costs was not significant $(P=0.717)$. In the second stage, 154 nonpregnant patients were divided into nine treatment groups, and the effects of changing TT to PAUT on the pregnancy rate, time-to-conception, and treatment costs were observed to be different from those of other treatments (all $P<0.05)$. Conclusion. Prodom-assisted urokinase can effectively treat male infertility secondary to impaired semen liquefaction.
\end{abstract}

\section{Introduction}

Impaired semen liquefaction (ISL) refers to the requirement of more than $1 \mathrm{~h}$ for semen liquefaction after ejaculation [1]. It is one of the commonest causes of male infertility and accounts for infertility in approximately $2.51 \%-$ $42.65 \%$ of males [2-5]. Currently, the pathogenesis of ISL is poorly understood. Several studies have suggested that ISL is caused by a decrease in plasminogen activators in the seminal plasma, such as urokinase and chymotrypsin, and their receptors [6-13]. Western and Chinese medicines are the primary methods used to treat ISL $[4,5,14-22]$. However, because of the complex metabolic mechanisms in the human body, neither oral Western nor Chinese medicines can resolve ISL in in vivo approaches $[4,5]$. Therefore, the focus of treatment has shifted toward improving ISL using in vitro approaches to identify an ideal therapeutic approach [14-19].

Of them, chymotrypsin and urokinase have been reported to improve ISL, method of using them varies, and, hence, the outcomes vary $[14,16-20]$. We previously used chymotrypsin to treat male infertility secondary to ISL using a special device-called "prodom"-that we designed, which yielded encouraging results [19]. Currently, prodom-assisted urokinase therapy in ISL has not been reported in clinical practice. Therefore, the purpose of this study was to evaluate the clinical efficacy of prodom in treating ISL-induced male infertility using urokinase therapy. 


\section{Materials and Methods}

2.1. Patients. In this randomized controlled trial study, consecutive data of 313 patients with ISL from January 1, 2012, to December 31, 2019, were collected from the Affiliated Hospital of Zunyi Medical University, China. The inclusion criteria were the following: complete clinical records (outpatient from January 1, 2012, to December 31, 2019) with follow-up of 9-96 months. The exclusion criteria were the following: (1) unimpaired semen liquefaction; (2) organic diseases, including uremia, cirrhosis and liver failure, lung failure, endocrine dysfunction, mental disorders, azoospermia, and severe oligospermia; (3) incomplete clinical records; or (4) termination of the treatment or refusal for follow-up visits. While 52 patients terminated the treatment prematurely, the remaining 261 completed the study. The Institutional Review Board of the Affiliated Hospital of Zunyi Medical University approved the study in January 2012, 2015 and 2018.

2.2. Experimental Group and Measures in Each Group. The 261 patients with ISL were divided into prodom-assisted urokinase treatment (PAUT) group $(n=91)$, syringe-assisted urokinase treatment (SAUT) group $(n=86)$, and traditional treatment (TT) group $(n=84)$. Randomization included assigning a number to each patient using the random number table according to the order of visit. The number was then divided by 3 , and patients with remainders of 0,1 , and 2 were assigned to the PAUT, SAUT, and TT group, respectively. The allocation was concealed using sealed envelopes to randomize the distribution. In each of the groups, pregnancy, time-to-conception, and treatment costs were evaluated in the first stage. If the first stage of treatment failed, other treatment methods were initiated instead and the patients were grouped according to the newer treatment method in the second stage. Conception and live birth rates were used as measures of treatment success, and the success and failure rates were calculated for each treatment as well as all treatments cumulatively (see Figure 1). Patients in the PAUT group were administered urokinase (Tianjin Biochemical Pharmaceutical Co. Ltd., China, batch No. 031802014). One milliliter of 10,000 IU urokinase was injected into the vagina of the partner through the prodom during intercourse, which was synchronously released with ejaculation. This helped urokinase to be mixed with the semen. In the SAUT group, $0.5 \mathrm{~mL}$ of urokinase (5,000 IU) was injected into the vagina using a 5milliliter syringe before the penis was inserted as well as after the penis was removed during intercourse. In the TT group, $1,000 \mathrm{mg}$ of vitamin $\mathrm{C}$ and $100 \mathrm{mg}$ of vitamin $\mathrm{E}$ were administered everyday along with $10 \mathrm{~mL}$ zinc gluconate and a spermatogenic tablet (containing a traditional Chinese medicine) administered thrice a day as supplements.

2.3. Prodom: Composition and Operational Process. Prodom is a type of auxiliary reproductive device that temporarily sticks on the penis that aids the male partner in injecting a drug into the vagina of female partner simultaneous with ejaculation such that the drug can be mixed with the semen in the vagina, thus improving the semen composition and contributing toward conception. The prodom described in this study was primarily composed of a polyurethane (PU) film and an injection catheter. It was coated with pressuresensitive adhesive on the inner side of the PU film [19]. Operating process was as follows: (1) before coitus, $1 \mathrm{~mL}$ of sterile saline and urokinase solution was injected with a syringe in the vagina, while the prodom was pasted onto the erect penis using the pressure-sensitive adhesive set on the PU film. (2) The urokinase injection through the wife into the vagina was synchronized with ejaculation, thereby blending it with the ejaculation in the vagina [19].

2.4. Steps for the Use of Prodom-Assisted Urokinase and Syringe-Assisted Urokinase. The specific steps have been described previously [19]. In this study, chymotrypsin was replaced with urokinase.

2.5. Clinical Data Extraction. The available data included semen parameters (volume, $\mathrm{pH}$, liquefaction time, and sperm density, motility, live rate, and morphology), duration of infertility, routine urine tests (leukocytes and red blood cells), prostatic enlargement, seminal vesicle enlargement, white blood cells in prostatic massage fluid, chronic prostatitis, history of chronic prostatitis, history of seminal vesiculitis, age of both partners, pregnancy, time-to-conception, and treatment costs. Semen parameters were measured by semiautomatic semen analyzer (BEION S3-3, BEIONMED ${ }^{\circledR}$, China).

2.6. Diagnosis Standards. ISL refers to a condition in which the liquefaction time exceeds $60 \mathrm{~min}$. Male infertility refers to the failure to conceive despite having regular intercourse without contraception a couple living together for more than 1 year and without female infertility. ISL and male infertility were diagnosed according to the Fifth Edition (2010) of the WHO laboratory test manual for human semen and spermcervical mucus interactions [1].

2.7. Follow-Up. The median follow-up time was 4.8 (range, $0.75-8$ ) years. All the patients were followed up via telephone and regular outpatient visits. The parameters recorded during follow-ups through telephone consultation included the health status, pregnancy status, and time of conception. Outpatient follow-ups included general physical examinations, routine blood and biochemical examinations, and analysis of semen quality.

2.8. Statistical Analyses. Statistical analyses were performed using SPSS v18 (SPSS Inc., Chicago, IL, USA). The selected characteristics (including the clinical parameters described above) were compared between the treatment groups using the chi-squared test for categorical variables and $t$-test and one-way analysis of variance (one-way ANOVA) for quantitative data. Numerical variables are presented as mean \pm standard deviation, and categorical variables are presented as percentages. $P<0.05$ was considered significant. 


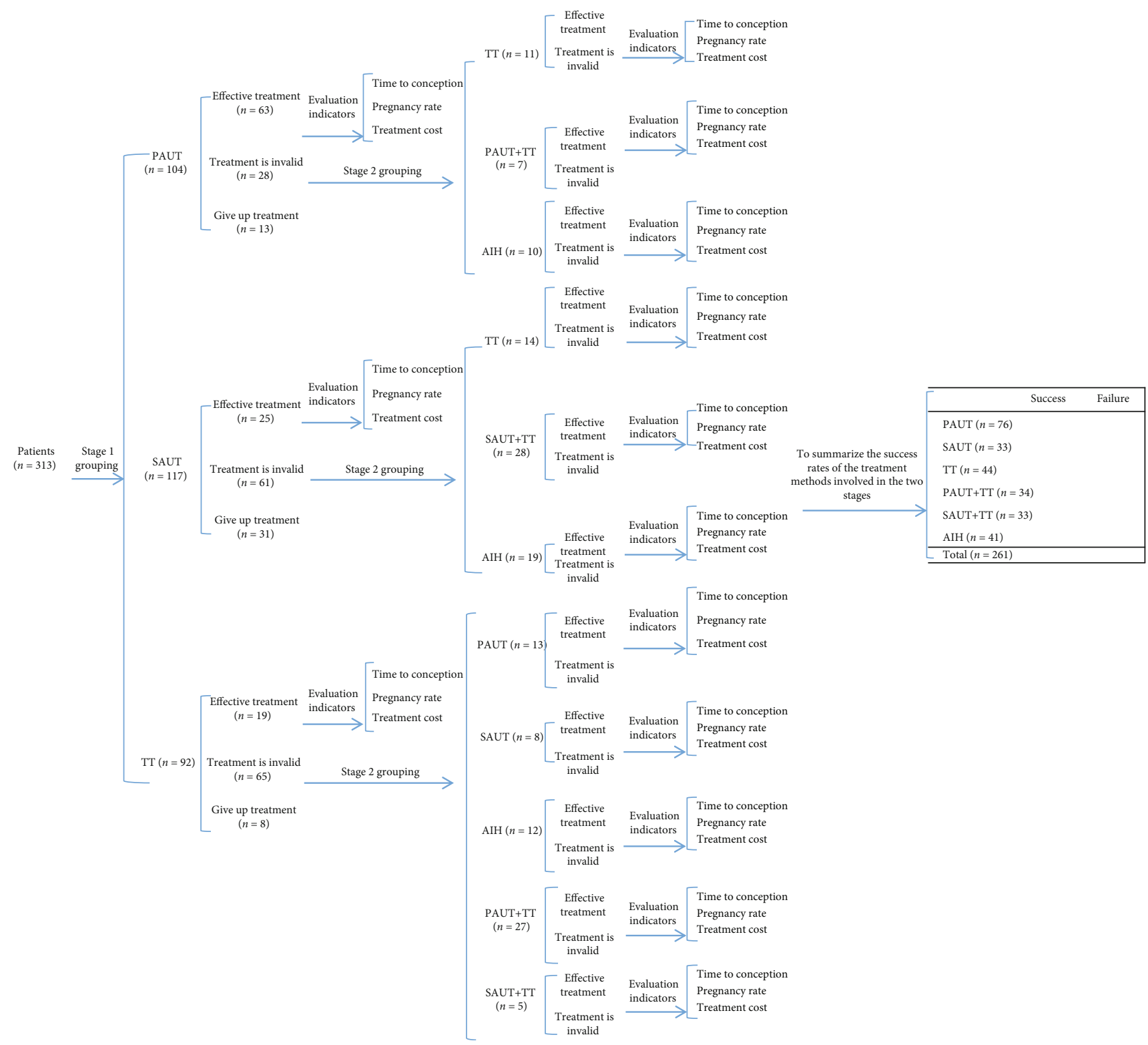

FIGURE 1: Flow chart of the experimental design.

\section{Results}

3.1. Baseline Characteristics. The duration since infertility was diagnosed was 1.5-21 years while the duration of infertility was $4.2 \pm 2.4$ years. The men were of $21-46$ years old (mean, 32.5 years), while their partners were 21-37 years old (mean, 27.5 years). Overall, 261 patients were divided into PAUT $(n=91)$, SAUT $(n=86)$, and TT $(n=84)$ groups. There were no significant differences between the groups in terms of semen parameters; duration of infertility; urine findings; prostatic enlargement; seminal vesicle enlargement; white blood cells in prostatic massage fluid, chronic prostatitis, or history of chronic prostatitis; history of seminal vesiculitis; and ages of the patients and their partners $(P>0.05)$ (Table 1). Of the 52 patients who terminated the treatment, the PAUT group accounted for $12.50 \%(13 / 104)$, SAUT group for $26.50 \%(31 / 117)$, and TT group for $8.70 \%(8 / 92)$ of them. The rate of abandoning treatment was lower in the PAUT group than those in the other two groups. The differences between the groups were statistically significant $(P=0.001)$.

3.2. Comparison of Pregnancy Rate, Time-to-Conception, and Treatment Costs in the First Stage of Treatment. In the first stage of treatment, in the PAUT, SAUT, and TT groups, As presented in Table 2, the pregnancy rate was $69.23 \%$ (63/91), 29.07\% (25/86), and 22.62\% (19/84), respectively; time-to-conception was $2.66 \pm 1.44$ months, $3.69 \pm 2.61$ months, and $3.86 \pm 3.00$ months, respectively; cost of treatment was $658.18 \pm 398.40,666.67 \pm 507.50$, and $680.56 \pm$ $480.94 \$$, respectively. The three treatment methods were not similar in terms of the pregnancy rate $\left(\chi^{2}=256.075\right.$ and $P<0.0001)$ and time-to-conception $(F=20.411$ and $P<0.0001)$; however, the difference in treatment cost 
TABLE 1: Baseline characteristics of clinical data in each group.

\begin{tabular}{|c|c|c|c|c|}
\hline Groups parameters & PAUT group $(n=91)$ & SAUT group $(n=86)$ & TT group $(n=84)$ & $P$ value \\
\hline Male's age (year) & $30.4 \pm 6.3$ & $31.9 \pm 6.3$ & $30.1 \pm 5.5$ & $0.103^{\dagger}$ \\
\hline Female's age (year) & $27.3 \pm 4.9$ & $28.6 \pm 4.6$ & $27.2 \pm 4.5$ & $0.097^{\dagger}$ \\
\hline \multicolumn{5}{|l|}{ Semen parameters } \\
\hline Emen volume (mL) & $3.46 \pm 0.89$ & $3.41 \pm 0.84$ & $3.49 \pm 0.78$ & $0.821^{\dagger}$ \\
\hline $\mathrm{pH}$ & $7.40 \pm 0.16$ & $7.44 \pm 0.17$ & $7.39 \pm 0.15$ & $0.084^{\dagger}$ \\
\hline Liquefaction time (min) & $>60$ & $>60$ & $>60$ & NS \\
\hline Sperm density $\left(\times 10^{6} / \mathrm{mL}\right)$ & $45.89 \pm 10.58$ & $45.35 \pm 9.28$ & $45.35 \pm 9.13$ & $0.912^{\dagger}$ \\
\hline \multicolumn{5}{|l|}{ Sperm motility, \% } \\
\hline a class & $18.89 \pm 3.29$ & $19.17 \pm 3.64$ & $18.69 \pm 3.31$ & $0.650^{\dagger}$ \\
\hline $\mathrm{a}+\mathrm{b}$ class & $42.45 \pm 5.52$ & $42.31 \pm 4.94$ & $42.94 \pm 5.71$ & $0.729^{\dagger}$ \\
\hline Live sperm rate, $\%$ & $56.27 \pm 8.88$ & $56.05 \pm 9.06$ & $55.33 \pm 8.85$ & $0.771^{\dagger}$ \\
\hline \multicolumn{5}{|l|}{ Sperm morphology, \% } \\
\hline Normal & $29.84 \pm 7.37$ & $29.77 \pm 7.44$ & $30.00 \pm 6.94$ & $0.977^{\dagger}$ \\
\hline Abnormal & $70.16 \pm 7.37$ & $70.23 \pm 7.44$ & $70.00 \pm 6.94$ & $0.977^{\dagger}$ \\
\hline Duration time of infertility (year) & $4.78 \pm 3.63$ & $4.78 \pm 2.89$ & $4.45 \pm 2.60$ & $0.725^{\dagger}$ \\
\hline \multicolumn{5}{|l|}{ Routine urine test } \\
\hline WBC, \% & & & & $0.611^{*}$ \\
\hline$(+)$ & $5.49(5 / 91)$ & $7.14(6 / 84)$ & $7.41(6 / 81)$ & \\
\hline$(-)$ & $94.51(86 / 91)$ & $92.86(78 / 84)$ & $92.59(75 / 81)$ & \\
\hline $\mathrm{RBC}, \%$ & & & & $0.781^{*}$ \\
\hline$(+)$ & $5.49(5 / 91)$ & $5.95(5 / 84)$ & $7.41(6 / 81)$ & \\
\hline$(-)$ & $94.51(86 / 91)$ & $94.05(79 / 84)$ & $92.59(75 / 81)$ & \\
\hline Prostatic enlargement, $\%$ & & & & $0.938^{*}$ \\
\hline Yes & $9.89(9 / 91)$ & $7.14(6 / 84)$ & $8.64(7 / 81)$ & \\
\hline No & $90.11(82 / 91)$ & $92.86(78 / 84)$ & $91.36(74 / 81)$ & \\
\hline Seminal vesicle enlargement, $\%$ & & & & $0.760^{*}$ \\
\hline Yes & $5.49(5 / 91)$ & $4.76(4 / 84)$ & $8.64(7 / 81)$ & \\
\hline No & $94.51(86 / 91)$ & $95.24(80 / 84)$ & $91.36(74 / 81)$ & \\
\hline Prostatic massage fluid, $\%$ & & & & $0.798^{*}$ \\
\hline WBC (+) & $6.59(6 / 91)$ & $7.14(6 / 84)$ & $8.64(7 / 81)$ & \\
\hline WBC (-) & $93.41(85 / 91)$ & $92.86(78 / 84)$ & $91.36(74 / 81)$ & \\
\hline Chronic prostatitis, $\%$ & & & & $0.688^{*}$ \\
\hline Yes & $12.09(11 / 91)$ & $7.14(6 / 84)$ & $8.64(7 / 81)$ & \\
\hline No & $87.91(80 / 91)$ & $92.86(78 / 84)$ & $91.36(74 / 81)$ & \\
\hline History of chronic prostatitis, $\%$ & & & & $0.577^{*}$ \\
\hline Yes & $4.40(4 / 91)$ & $8.33(7 / 84)$ & $7.41(6 / 81)$ & \\
\hline No & $95.60(87 / 91)$ & $91.67(77 / 84)$ & $92.59(75 / 81)$ & \\
\hline History of seminal vesiculitis, \% & & & & $0.993^{*}$ \\
\hline Yes & $4.40(4 / 91)$ & $4.76(4 / 84)$ & $4.94(4 / 81)$ & \\
\hline No & $95.60(87 / 91)$ & $95.24(80 / 84)$ & $95.06(77 / 81)$ & \\
\hline Give up treatment, $\%$ & $12.50(13 / 104)$ & $26.50(31 / 117)$ & $8.70(8 / 92)$ & $0.001^{*}$ \\
\hline
\end{tabular}

Abbreviations: PAUT: prodom-assisted urokinase treatment; SAUT: syringe-assisted urokinase treatment; TT: traditional treatment. $P$ values were calculated using ${ }^{\dagger}$ one-way analysis of variance (one-way ANOVA) and *chi-squared; boldface text represents statistical significance.

( $F=0.333$ and $P=0.717$ ) was not significant. Of them, the effects of PAUT on pregnancy rate and time-to-conception were different than those of SAUT and TT $(P<0.05)$. There- fore, it can be considered that PAUT is superior to SAUT and TT in increasing the pregnancy rate and shortening the timeto-conception. 
TABLE 2: Pregnancy rate/time to conception/treatment cost between groups were compared in the first stage.

\begin{tabular}{|c|c|c|c|c|c|c|}
\hline $\begin{array}{l}\text { Groups } \\
\text { Parameters }\end{array}$ & PAUT group $(n=91)$ & SAUT group $(n=86)$ & TT group $(n=84)$ & Total $(n=261)$ & $x^{2} / F$ & $P$ value \\
\hline Pregnancy rate, $\%$ & $69.23(63 / 91)$ & $29.07(25 / 86)$ & $22.62(19 / 84)$ & $41.00(107 / 261)$ & 256.075 & $<0.0001^{*}$ \\
\hline Time to conception (months) & $1.44 \pm 2.66$ & $3.69 \pm 2.61$ & $3.86 \pm 3.00$ & $3.02 \pm 2.96$ & 20.411 & $<0.0001^{\dagger}$ \\
\hline Treatment cost $(\$)$ & $658.18 \pm 398.40$ & $666.67 \pm 507.50$ & $680.56 \pm 480.94$ & $692.56 \pm 416.03$ & 0.333 & $0.717^{\dagger}$ \\
\hline
\end{tabular}

Abbreviations: PAUT: prodom-assisted urokinase treatment; SAUT: syringe-assisted urokinase treatment; TT: traditional treatment. $P$ values were calculated using *chi-squared and ${ }^{\dagger}$ one-way analysis of variance (one-way ANOVA). In the first stage of treatment, the three treatment methods were not the same in terms of the influence of pregnancy rate $\left(\chi^{2}=256.075\right.$ and $\left.P<0.0001\right)$ and time to conception $(F=20.411$ and $P<0.0001)$, and the difference in treatment cost $(F=0.333$ and $P=0.717)$ was not significant. Among them, the effect of PAUT on pregnancy rate and time to conception was different from that of SAUT and TT (all $P<0.05$ ). It can be considered that the PAUT is superior to the SAUT and the TT in increasing the pregnancy rate and shortening the time to conception. The boldface represents statistical significance.

3.3. Pregnancy Rate, Time-to-Conception, and Treatment Costs between Groups in the Second Stage of Treatment. After 9 months, 154 patients who were unable to achieve pregnancy in the first stage of treatment in PAUT $(n=28)$, SAUT $(n=61)$, and TT groups $(n=65)$ were assigned to receive other treatments in the second stage. As presented in Table 3, the pregnancy rate was $36.36 \%$ (4/11), timeto-conception was $19.50 \pm 5.84$ months, and cost of treatment was \$2,538 \pm 179 in the patients in PAUT group who were switched to TT group. The pregnancy rate was $50 \%$ (17/34), time-to-conception was $17.75 \pm 6.02$ months, and cost of treatment was $\$ 4,464 \pm 177$ in the PAUT group combined with the TT group. The pregnancy rate was $70 \%$ (7/10), time-to-conception was $21.40 \pm 16.73$ months, and cost of treatment was $\$ 19,621 \pm 6,164$ in the patients in PAUT group who underwent artificial insemination with husband's semen (AIH). The pregnancy rate was $42.11 \%$ (8/19), time-to-conception was $17.86 \pm 5.91$ months, and cost of treatment was $\$ 2,597 \pm 223$ in the patients in SAUT group who were switched to TT group. The pregnancy rate was $51.52 \%$ (17/33), time-to-conception was $13.83 \pm 7.21$ months, and cost of treatment was $\$ 2,248 \pm 706$ in the SAUT group combined with the TT group. The pregnancy rate was $42.11 \%(8 / 19)$, time-to-conception was $19.84 \pm$ 15.90 months, and cost of treatment was $\$ 18,586 \pm 4,237$ in the patients in SAUT group who underwent AIH. The pregnancy rate was $69.23 \%$ (9/13), time-to-conception was $12.67 \pm 4.40$ months, and cost of treatment was $\$ 1,358 \pm$ 266 in the patients in TT group who were switched to PAUT group. The pregnancy rate was $75.00 \%(6 / 8)$, timeto-conception was $16.62 \pm 4.70$ months, and cost of treatment was \$2,129 \pm 262 in the patients in TT group who were switched to SAUT group. The pregnancy rate was $66.67 \%$ (8/12), time-to-conception was $21.83 \pm 18.75$ months, and cost of treatment was $\$ 20,726 \pm 3,336$ in the patients in TT group who underwent AIH. The effects on pregnancy rate $\left(\chi^{2}=8.405\right.$ and $\left.P=0.015\right)$, time-toconception $(F=39.876$ and $P<0.0001)$, and cost of treatment $(F=129.567$ and $P<0.0001)$ of the nine treatments mentioned above were not similar to each other. Furthermore, in these treatment groups, the effects of switching from TT to PAUT on the pregnancy rate, time-to-conception, and cost of treatment were different from those of the other treatments (all $P<0.05$ ). Therefore, our results demonstrated that switching from TT to PAUT was superior to the other treatments in the pregnancy rate, timeto-conception, and cost of treatment.

3.4. Analysis of the Success Rates of Different Treatments. We summarized the treatment methods, including PAUT, SAUT, TT, PAUT+TT, SAUT+TT, and AIH. We used the conception and live birth rates as measures of the treatment success and calculated the success and failure rates for each treatment as well as the overall success and failure rates for all the treatments. As presented in Table 4, we sorted the statistics of the efficacies of these six treatment methods. Of them, PAUT had a $94.74 \%(72 / 76)$ success rate and $5.26 \%$ (4/76) failure rate; SAUT had $87.88 \%$ (29/33) success rate and $12.12 \%(4 / 33)$ failure rate; TT had $65.91 \%(29 / 44)$ success rate and $34.09 \%$ (15/44) failure rate; PAUT+TT had success and failure rates of $50.00 \%$ (17/34) each; SAUT+TT had $51.52 \%(17 / 33)$ success rate and $48.48 \%(16 / 33)$ failure rate; AIH had 56.10\% (23/41) success rate and 43.09\% (18/41) failure rate. The overall treatment success rate was $71.65 \%$ (187/261), and the failure rate was $28.35 \%$ (74/261). Chisquare check was used for Row $\mathrm{x}$ List, and the success rates of the above six treatments were not the same $\left(\chi^{2}=38.213\right.$ and $P<0.0001)$. Furthermore, of them, PAUT was different from other groups in pairwise comparisons (all $P<0.05$ ). These results indicate that the success rate of PAUT was higher than those of the other treatments.

\section{Discussion}

Currently, there are several methods to treat ISL, including oral administration, muscle injection, transurethral administration, transrectal administration, transvaginal administration, and acupuncture. All these methods have been reported to be effective although they have their own limitations; consequently, satisfactory results are not achieved in some patients. Previous studies have confirmed that the addition of chymotrypsin to the semen after ejaculation could completely correct ISL and improve sperm vitality without damaging the sperm quality. We used a syringe/prodom to assist the injection of chymotrypsin into the vagina during coitus to treat ISL, which produced better results. Further, it was previously reported that the effects of treatment using prodom-assisted chymotrypsin in the 


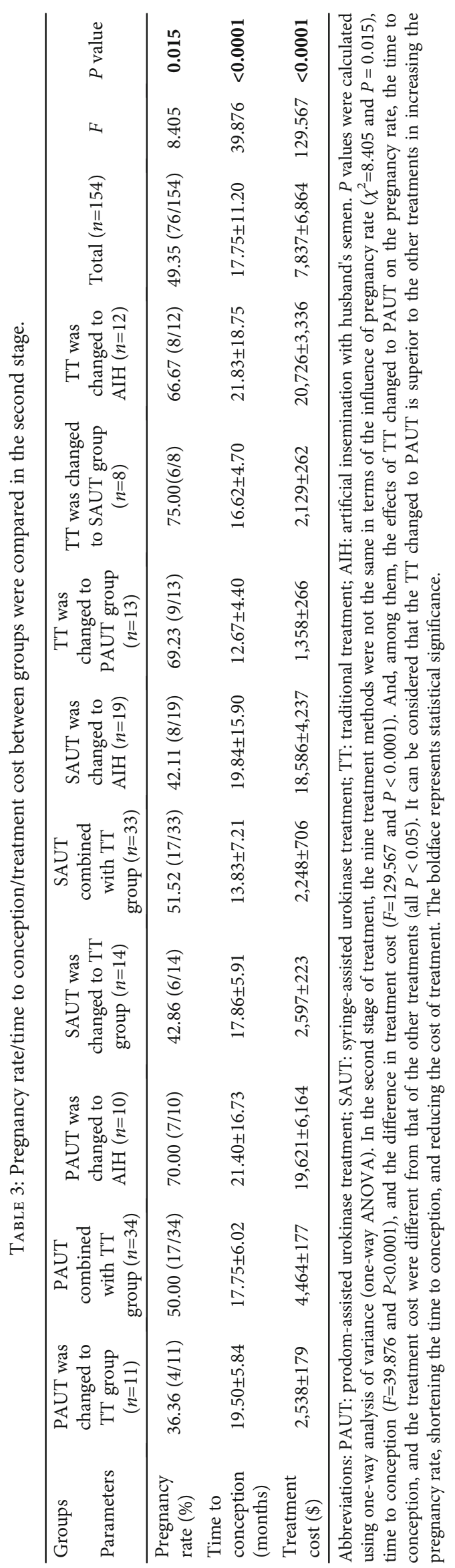


TABLE 4: Compare the efficacy of different treatments in this study.

\begin{tabular}{|c|c|c|c|c|c|}
\hline \multirow{2}{*}{ Treatments } & \multicolumn{2}{|c|}{ Therapeutic efficiency } & \multicolumn{2}{|c|}{ Therapeutic inefficiency } & \multirow{2}{*}{ Total } \\
\hline & $n 1$ & $\%$ & $n 2$ & $\%$ & \\
\hline PAUT & 72 & $94.74(72 / 76)^{\Delta \Phi \Sigma}$ & 4 & $5.26(4 / 76)$ & 76 \\
\hline SAUT & 29 & $87.88(29 / 33)^{\Delta \Phi \Sigma}$ & 4 & $12.12(4 / 33)$ & 33 \\
\hline $\mathrm{TT}$ & 29 & $65.91(29 / 44)^{\Delta}$ & 15 & $34.09(15 / 44)$ & 44 \\
\hline PAUT+TT & 17 & $50.00(17 / 34)^{\Delta}$ & 17 & $50.00(17 / 34)$ & 34 \\
\hline SAUT+TT & 17 & $51.52(17 / 33)^{\Delta}$ & 16 & $48.48(16 / 33)$ & 33 \\
\hline $\mathrm{AIH}$ & 23 & $56.10(23 / 41)^{\Delta}$ & 18 & $43.90(18 / 41)$ & 41 \\
\hline Total & 187 & $71.65(187 / 261)$ & 74 & $28.35(74 / 261)$ & 261 \\
\hline
\end{tabular}

Abbreviations: PAUT: prodom-assisted urokinase treatment; SAUT: syringe-assisted urokinase treatment; TT: traditional treatment; AIH: artificial insemination with husband's semen. The chi-square check is used for Row x List, and the efficacy of the above six treatment regimens is not all the same $\left(\chi^{2}=38.213\right.$ and $\left.{ }^{\Delta} P<0.0001\right)$. Among them, there was no significant difference in the efficacy of PUAT and SAUT $\left(\chi^{2}=0.270\right.$ and $\left.{ }^{\Phi} P=0.550\right)$, but there was difference between them and other treatments (all $\left.{ }^{\Sigma} P<0.05\right)$. It can be considered that the efficacy of PAUT and SAUT was higher than that of the other treatments.

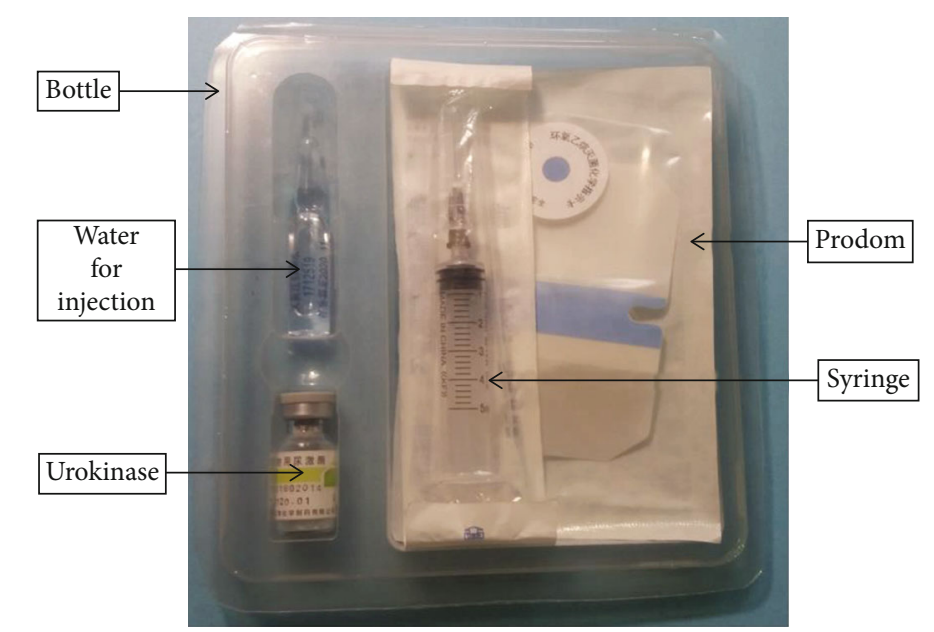

Figure 2: Packing sample product label of prodom-assisted urokinase.

vagina were better than those of using a syringe to inject chymotrypsin in the vagina [19]. However, PAUT has not been reported previously.

In this study, we evaluated the clinical efficacy of prodom-assisted urokinase in treating ISL. The results demonstrated that the pregnancy rate was $69.23 \%$ in the PAUT group, which was higher than those of both the SAUT (29.07\%) and TT (22.62\%) groups. Time-to-conception was $2.66 \pm 1.44$ months, which was significantly lower than those in both the SAUT $(3.69 \pm 2.61$ months $)$ and TT $(3.86 \pm 3.00$ months) groups in the first stage (all $P<0.05)$. However, the cost of treatment in the PAUT, SAUT, and TT groups was $658.18 \pm 398.40,666.67 \pm 507.50$, and 680.56 \pm 480.94 \$, respectively, which was not significantly different $(P=0.717)$. However, in the second stage, after switching from TT to PAUT, the effects on the pregnancy rate, timeto-conception, and cost of treatment were significantly different from those of the other treatments (all $P<0.05$ ). Consequently, these results prove that PAUT is superior to other treatments in increasing the pregnancy rate, shortening the time-to-conception, and reducing the cost of treatment.

According to the results of this study, in the first stage of treatment, PAUT was superior to SAUT and TT in increas- ing the pregnancy rate and shortening the time-toconception (Table 2). In the second stage of treatment, changing TT to PAUT was superior to the other treatments in increasing the pregnancy rate, shortening the time-to-conception, and reducing the cost of treatment (Table 3).

Regarding the overall efficacy and specific efficacy of different treatments used in ISL, we summarized the six treatment methods of PAUT, SAUT, TT, PAUT+TT, SAUT+TT, and AIH. The overall treatment success rate was $71.65 \%$, and the treatment success rate of PAUT, SAUT, and TT was $94.74 \%, 87.88 \%$, and $65.91 \%$, respectively (Table 4). The treatment success rates of PAUT and SAUT were higher than that of TT and the overall treatment success rate. Additionally, the specific success rates of these six treatment regimens were not similar $\left(\chi^{2}=38.213\right.$ and $P<$ $0.0001)$. Of them, PAUT was different from other groups in pairwise comparisons (all $P<0.05$ ). Therefore, the results prove that the efficacy of PAUT was higher than those of the other treatment regimens.

Previous studies have reported that the pregnancy rate obtained by treating ISL using Chinese medicines was $38.71 \%$ [4], while that obtained with the use of chymotrypsin was $21 \%-53.21 \%[18,19]$. The pregnancy rate reported was 
69.23\% using PAUT in this study, which is higher than those in previous reports. Furthermore, in terms of time-toconception and cost of treatment, PAUT was also reported to be superior to other treatments in this study and those in previous reports. Additionally, complications, such as allergies, genital tract injuries, bleeding, and infections, were not observed with this treatment regimen. Therefore, we recommend the use of PAUT in treating ISL (Figure 2).

However, the present study had several limitations. The overall pregnancy rate was $71.65 \%$ (187/261), which implied that $28.35 \%(74 / 261)$ of patients did not benefit from the treatment regimens used in this study. Therefore, new treatment methods and treatment policies should be further explored [21-25]. We are working toward transferring the patients with response failure for the first two stages of treatment to the third stage of treatment and use a rotation model with the aforementioned treatments to improve the outcomes. Furthermore, this study was conducted only in the Chinese population, which included a majority of Han Chinese population but only a small proportion of Hui, Miao, Buyi, and Gelao populations who have different lifestyles. Our study did not subdivide the participants based on such populations. Additionally, this study included a relatively small number of patients from a single center. These limitations may have affected the results and conclusions. Therefore, larger and multicenter studies are necessary to corroborate our findings.

\section{Conclusion}

In conclusion, the prodom can increase the rate of pregnancy, shorten the time-to-conception, and reduce cost of treatment in patients with ISL by the virtue of assisting in the administration of urokinase into the vagina.

\section{Data Availability}

The data used to support the findings of this study are included within the article.

\section{Conflicts of Interest}

The authors have no conflicts to disclose.

\section{Authors' Contributions}

Kaiyi Mao, Tong Liu, Mengzhi Li, Zidong Zhou, Chao Chen, Chengren Gou, Yuhong Yao, Chenghong Zou, and Xu Li participated in protocol/project development and data collection. Kaiyi Mao, Zidong Zhou, Chengren Gou, and Zongping Chen participated in data summarization, data analysis, and drafting the manuscript. Yong Yan formed the project development. Kaiyi Mao, Chengren Gou, and Zidong Zhou are considered co-first authors.

\section{Acknowledgments}

This study was supported by funding from Science and Technology Project of Honghuagang District, Zunyi City [No. Industrial Class (2016) 14], Special Fund for Governor of Guizhou Province (No. 51010106), Guizhou Science and Technology Department Fund (No. LKZ [2011]07), and Department of Health of Guizhou Province Fund (No. 20120424). The authors would like to thank Professor Zhou Yuanzhong (School of Public Health Management, Zunyi Medical University) for the guidance with statistics. The authors wish to acknowledge the efforts of the participants and investigators of the study. At the same time, we would like to thank Editage (http://www.editage.com) for English language editing.

\section{References}

[1] World Health Organization, WHO Laboratory Manual for the Examination and Processing of Human Semen, World Health Organization, Geneva, 5th edn edition, 2010.

[2] M. L. Eisenberg, R. B. Lathi, V. L. Baker, L. M. Westphal, A. A. Milki, and A. K. Nangia, "Frequency of the male infertility evaluation: data from the National Survey of Family Growth," Journal of Urology, vol. 189, no. 3, pp. 1030-1034, 2013.

[3] A. J. Schaeffer and J. M. Hotaling, "Claims-based analysis of male infertility: a cautious step in the right direction," Fertility and Sterility, vol. 105, no. 3, pp. 599-600, 2016.

[4] G.-B. Xiong, W.-L. Yao, and F.-H. Wu, "Shenfu Qiangjing decoction improves non-inflammatory and non-liquefied semen in kidney-yang deficiency men," Zhonghua Nan Ke Xue., vol. 15, no. 12, pp. 1138-1141, 2009.

[5] J. H. Check, "Treatment of male infertility," Clinical and Experimental Obstetrics \& Gynecology, vol. 34, no. 4, pp. 201-206, 2007.

[6] A. Arnaud, J.-F. Schved, J.-C. Gris, P. Costa, H. Navratil, and C. Humeau, "Tissue-type plasminogen activator level is decreased in human seminal plasma with abnormal liquefaction," Fertility and Sterility, vol. 61, no. 4, pp. 741-745, 1994.

[7] C. Y. Hong, B. N. Chiang, J. J. Huang, and P. Wu, "Two plasminogen activators, streptokinase and urokinase stimulate human sperm motility," Andrologia, vol. 17, no. 4, pp. 317320, 1985.

[8] B. Astedt, P. Wallén, and B. Aasted, "Occurrence of both urokinase and tissue plasminogen activator in human seminal plasma," Thrombosis Research, vol. 16, no. 3-4, pp. 463-472, 1979.

[9] H. Xunbin, X. Wenjia, X. Chengliang, X. Dunzhen, S. Jiyun, and Z. Jieling, "Studies on the relationship between urokinase plasminogen activator (uPA) and human sperm motility," Journal of Tongji Medical University, vol. 17, no. 4, pp. 213217, 1997.

[10] K. K. Vihko, T. L. Penttila, M. Parvinen, and D. Belin, "Regulation of urokinase- and tissue-type plasminogen activator gene expression in the rat seminiferous epithelium," Molecular Endocrinology, vol. 3, no. 1, pp. 52-59, 1989.

[11] C. Li, J. Zhang, Y. Jiang, V. Gurewich, Y. Chen, and J. N. Liu, "Urokinase-type plasminogen activator up-regulates its own expression by endothelial cells and monocytes via the u-PAR pathway," Thrombosis Research, vol. 103, no. 3, pp. 221-232, 2001.

[12] N. Montuori, A. Mattiello, A. Mancini et al., "Urokinase-type plasminogen activator up-regulates the expression of its cellular receptor through a post-transcriptional mechanism," FEBS Letters, vol. 508, no. 3, pp. 379-384, 2001.

[13] F. Odet, R. Guyot, P. Leduque, and B. Le Magueresse-Battistoni, "Evidence for similar expression of protein $\mathrm{C}$ inhibitor 
and the urokinase-type plasminogen activator system during mouse testis development," Endocrinology, vol. 145, no. 3, pp. 1481-1489, 2004.

[14] M. L. Check, D. Katsoff, J. H. Check, and D. Summers-Chase, "Effect of treating sperm with low hypo-osmotic swelling test scores with chymotrypsin on pregnancy rates after conventional in vitro fertilization- embryo transfer," Fertility and Sterility, vol. 82, no. 3, pp. 741-742, 2004.

[15] S. Peng, Y. Zheng, K. Zheng et al., "Effect of a comprehensive therapy plus gushenyutai plaster administered at guanyuan (CV 4) on male infertility associated with semen non-liquefaction," Journal of Traditional Chinese Medicine, vol. 34, no. 6, pp. 666-672, 2014.

[16] M. L. Check, D. Kiefer, J. H. Check, W. Hourani, and R. Long, "Treatment of sperm with subnormal host scores with chymotrypsin/viable pregnancy after IUI," Archives of Andrology, vol. 48, no. 2, pp. 155-158, 2009.

[17] K. L. Honea, V. L. Houserman, D. C. Merryman, D. A. Free, and S. E. Stringfellow, "Effect of limited proteolysis with alpha-chymotrypsin on semen with an abnormal sperm penetration assay and possible application for in vitro fertilization or intrauterine insemination," Journal of Assisted Reproduction and Genetics, vol. 10, no. 4, pp. 255-260, 1993.

[18] C. Freischem, J. Bordt, J. Hanker, H. Schneider, and E. Nieschlag, "Pregnancy after treatment of ejaculate with alpha-chymotripsine because of failure to liquefy," Geburtshilfe und Frauenheilkunde, vol. 43, no. 8, pp. 490-491, 1983.

[19] Z. Chen, T. Song, Y. Yan et al., "Prodom: a new assisted reproductive device to treat male infertility caused by impaired semen liquefaction," International Journal of Clinical and Experimental Medicine, vol. 12, no. 4, pp. 4088-4099, 2019.

[20] J. C. Martinez-Soto, J. Landeras, M. Mollá et al., “Total urokinase-type plasminogen activator (uPA) levels in seminal plasma are associated with positive assisted reproductive technology outcomes," Journal of Assisted Reproduction and Genetics, vol. 35, no. 6, pp. 1091-1101, 2018.

[21] M. P. Bitler and L. Schmidt, "Utilization of infertility treatments: the effects of insurance mandates," Demography, vol. 49, no. 1, pp. 125-149, 2012.

[22] Ministry of Health and long-term care, "Improving access to safe fertility treatments; 10 April, 2014," October 2015, http://www.news.ontario.ca/mohltc/en/2014/04/improvingaccess-to-safe-fertility-treatments.html.

[23] "Québec assisted reproduction program," Octoer 2015, http:// www.sante.gouv.qc.ca/en/programmes-et-mesures-daide/ programme-quebecois-de-procreation-assistee/description/.

[24] J. M. Dupree, "Insurance coverage for male infertility care in the United States," Asian Journal of Andrology, vol. 18, no. 3, pp. 339-341, 2016.

[25] A. Chandra, C. E. Copen, and E. H. Stephen, "Infertility service use in the United States: data from the National Survey of Family Growth, 1982-2010," in National health statistics reportsNational Center for Health Statistics, Hyattsville, MD, USA. 